\title{
Experimental validation of fracture aperture determination from borehole electric microresistivity measurements
}

\author{
Michel Ponziani ${ }^{1}$, Evert Slob ${ }^{1}$, Stefan Luthi ${ }^{2}$, Richard Bloemenkamp ${ }^{3}$, and Isabelle Le Nir ${ }^{3}$
}

\begin{abstract}
We studied the electric response of fractures with laboratory experiments and numerical simulations for a full-bore formation microimaging tool. The laboratory setup was designed and built to perform controlled experiments with accurate measurements of all principal properties involved for electric borehole imaging. These properties are formation resistivity, mud resistivity, fracture aperture, pad position, and button current. The experiments were conducted on two types of limestone for fracture apertures ranging from 0.1 to $0.9 \mathrm{~mm}$ and mud/formation resistivity contrasts varying from $1 / 100$ to $1 / 10,000$. A numerical model was used to reproduce the laboratory configuration and to validate the results. The model proved to be an effective tool to optimize the experimental setup, and it was also used to study the effect of standoff (up to $5 \mathrm{~mm}$ ) on the measured integrated additional current. Linear relationships between the fracture aperture and measured integrated current were found to be valid for the laboratory experiment and the corresponding numerical simulation. The measured integrated current could therefore be used to determine the fracture aperture if the other parameters are known. Two coefficients in the relationship were found to differ from those previously found using numerical simulations for the actual borehole situation. These differences are attributed to tool- and scale-dependent factors.
\end{abstract}

\section{INTRODUCTION}

Natural fractures have an important influence on reservoir productivity. For ideal fractures with perfectly smooth parallel faces, the volume flow rate is proportional to the cube of the fracture aper- ture (e.g., Gangi, 1978). Therefore, changes in the fracture aperture result in substantial changes in the potential well productivity, and the determination of fracture apertures in the borehole is thus of paramount importance in fractured reservoirs. However, real fractures have a certain tortuosity that extends the fluid path causing a deviation from the cubic law (e.g., Gangi, 1978; Brown and Scholz, 1986), with the actual flow rate between rough surfaces being about $70 \%$ to $90 \%$ of that predicted by the parallel plate model (Brown, 1987). Information about fractures is also relevant during well construction because fractures can cause borehole washouts, lost circulation problems (i.e., uncontrolled flow of whole mud into a formation), and potentially the loss of wells (Bratton et al., 2006).

The full-bore Formation MicroImager (FMI - mark of Schlumberger) is a borehole electric imaging tool that characterizes sedimentary and tectonic structures in boreholes, including fractures (Luthi, 2001). This device is an evolution of the Formation MicroScanner (FMS - mark of Schlumberger) tool (Ekstrom et al., 1987), with a higher lateral and vertical resolution and improved borehole coverage. Typically, the FMI is operated in water-based mud because it relies on conductive paths from the emitting electrodes to the return electrodes, but a newer version has been modified for applications in oil-based mud (Laronga et al., 2011). The measurements are performed by arrays of electrodes mounted on a pad, which is held at a known potential with respect to a return electrode in the upper part of the tool. Currents emitted from these electrodes are recorded at a high-sampling rate and are used to produce conductance images of the borehole walls (1-cm image resolution in the vertical and azimuthal directions). Images from the tool in water-based mud show open fractures as conductive features because the emitted current increases in front of the fractures due to invasion of conductive drilling mud (Pezard and Luthi, 1988). The images can also be used to interpret the geometry of breakouts and determine the in situ stress direction (Rajabi et al., 2010). The properties affecting the electric response of fractures are their aperture, the resistivity contrast between the frac-

Manuscript received by the Editor 21 July 2014; revised manuscript received 9 December 2014; published online 12 March 2015.

${ }^{1}$ Delft University of Technology, Department of Geoscience and Engineering, Delft, The Netherlands. E-mail: m.ponziani@tudelft.nl; e.c.slob@tudelft.nl.

${ }^{2}$ Delft University of Technology, Department of Geoscience and Engineering, Delft, The Netherlands and Schlumberger Limited, The Hague, The Netherlands. E-mail: s.m.luthi@tudelft.nl.

${ }^{3}$ Études et Productions Schlumberger, Clamart, France. E-mail: rbloemenkamp@ @lb.com; lenir@slb.com.

(C) 2015 Society of Exploration Geophysicists. All rights reserved. 
ture and the formation, the dip angle (i.e., the angle between the steepest line in the fracture plane and the horizontal, measured in the vertical plane), and the tool standoff (the distance between the tool and the borehole wall). Luthi and Souhaité (1990) investigate the electric response of an FMS tool with a 3D finite-element model and derive a semiempirical formula to estimate the fracture aperture based on the other relevant parameters, but the formula has not been validated with laboratory experiments thus far.

In this paper, we present a laboratory setup to perform electric measurements with an actual FMI pad under controlled conditions. Experiments were performed on Irish limestone blocks for a wide range of fracture apertures, mud resistivities, and standoffs. The fracture properties were acquired using a digital microscope, a conductivity meter, and a four-electrode resistivity device. A 3D numerical modeling code was used to model this laboratory setup and to validate the experimental results.

\section{MODELING}

The response of an FMI tool to an open (conductive) fracture is described by the relationship found by Luthi and Souhaité (1990) as

$$
W=c A R_{m}^{b} R_{x o}^{1-b},
$$

where the fracture aperture or width $W$ is expressed as a function of the integrated additional current $A$, the resistivity of the formation $R_{x o}$, and the resistivity of the mud $R_{m}$, through the tool-dependent coefficients $b$ and $c$. The current emitted by a single button in front of a fracture was simulated with a 3D finite-element modeling code. The fracture is modeled as a thin sheet of uniform resistivity corresponding to the mud resistivity, and it is assumed to be planar, with parallel faces, and of infinite extent.

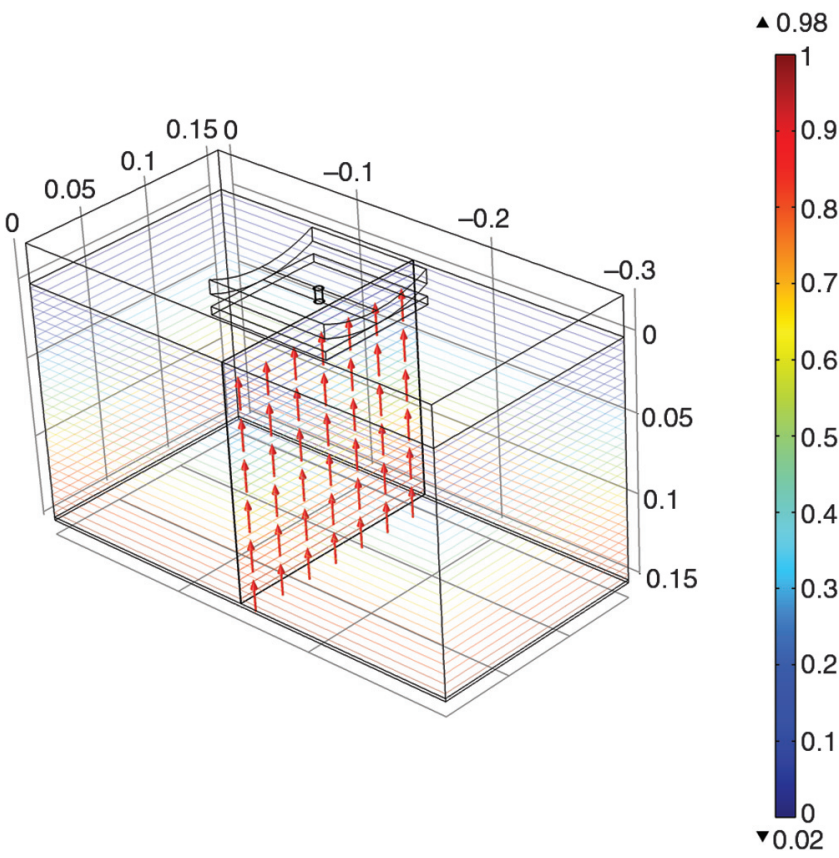

Figure 1. Numerical simulations of a laboratory-scale sample showing the stationary solutions of the electric potential distribution in the sample (from $1 \mathrm{~V}$ held at the return electrode to $0 \mathrm{~V}$ at the grounded pad) and the current density vectors (red arrows).
The value of $A$ caused by the higher conductivity of the fracture represents the amount of additional current injected into the formation divided by the voltage. It is calculated from the relationship

$$
A=\frac{1}{V_{e}} \int_{z_{0}}^{z_{n}}\left\{I_{b}(z)-I_{b m}\right\} \mathrm{d} z,
$$

where $V_{e}$ is the potential difference (in voltage) between the pad and the return electrode; $I_{b}$ is the button current (in $\mu \mathrm{A}$ ) as a function of the pad position $(z)$ across the fracture (with positions $z_{0}$ and $z_{n}$ being the first and last positions, respectively, at which the measurement is affected by the fracture); and $I_{b m}$ is the background current level (i.e., the button current in the undisturbed matrix below and above the fracture).

The model was obtained for a wide range of formation resistivities $R_{x o}=10$ to $\left.1000 \Omega \mathrm{m}\right)$ and fracture apertures $(W=50$ to $200 \mu \mathrm{m})$, with a fixed value of mud resistivity $\left(R_{m}=0.1 \Omega \mathrm{m}\right)$. Tool standoffs (up to $2.5 \mathrm{~mm}$ ) and fracture dips (from $0^{\circ}$ to $40^{\circ}$ ) were also investigated and were found to have an insignificant effect on equation 1 within the investigated ranges. Coefficient $b$ is primarily influenced by the amount of current focusing as a function of the resistivity contrast and the borehole diameter, whereas coefficient $c$ is a function of the resistivity contrast, the borehole diameter, and the square of the button diameter. Both coefficients were obtained numerically by fitting equation 1 to the results of numerical simulations at the borehole scale and were published as $b=0.863$ and $c=4.801 \mathrm{~mm}^{-1}$ (Luthi and Souhaité, 1990). Equation 1 is valid for other, similar tools, but the numerical values of $b$ and $c$ depend on the tool configuration.

\section{Numerical simulations of the laboratory setup}

Equation 1 was derived through numerical simulations that modeled the electric response of the tool in the borehole. To validate the relationship with experiments, a laboratory-scale setup must be realized. For this, a finite-element model of the experiments is necessary to link the small-scale setup to the borehole-scale situation. Moreover, the numerical model is needed to determine the proper configuration of the setup (e.g., sample size, position of the return electrode, current paths) and to validate the experimental results for different values of $W, R_{x o}$, and $R_{m}$, as well as standoff.

For this purpose, first a 3D numerical model of the laboratory measurements was designed. Two blocks of rock were designed and separated by a fracture represented by a thin sheet of uniform resistivity and parallel, planar faces. The tool pad was designed based on its real dimensions, with a curvature radius of $108 \mathrm{~mm}$ and one circular metal electrode surrounded by an insulating ring. The dimension of the electrode determines the amount of current measured; therefore, it is a crucial value in the determination of the integrated additional current. The metal electrode diameter is $4 \mathrm{~mm}$, but often half of the insulation is included, leading to a 5-mm electrode. The pad scans the rocks on one side, crossing the fracture perpendicularly on its way. The return electrode is a boundary condition covering the opposite side of the rocks. A constant voltage is applied on the return electrode, whereas the pad (including the measuring button) is grounded $(V=0)$. The model is discretized with an unstructured tetrahedral mesh using smaller elements along the fracture and the pad. Solutions are computed for the electric current equations under stationary conditions (Figure 1). 
Several parameters of the model can be changed to study the electric response for different setup and sample configurations, such as the size of the blocks, the applied voltage, the fracture aperture, and the mud resistivity. Numerical simulations were run to simulate the effect of $W$ and $R_{m}$ on the electric response of the planned laboratory-scale sample, consisting of two blocks of $150 \times 150 \times$ $150 \mathrm{~mm}$ separated by a thin fracture, with the purpose of determining the validity of equation 1 at the laboratory scale. Different fracture apertures were considered, from $100 \mu \mathrm{m}$ to $1 \mathrm{~mm}$. The formation resistivity, which corresponds to the near-wellbore part of the rock invaded by the drilling fluid, was fixed at $2400 \Omega \mathrm{m}$, which is the resistivity of the Irish limestone used for the experiments. The mud resistivity varied from 0.24 to $24 \Omega \mathrm{m}$, leading to resistivity contrasts from $1 / 100$ to $1 / 10,000$. Solutions were obtained for different positions of the pad along the $z$-axis, which is perpendicular to the fracture trace with the origin in the center of the fracture. The effect of standoff was also studied with numerical simulations, and the results showed that the button currents decrease in their peaks but widen in their shape with increasing standoff. However, the overall value of $A$ seems to be independent of standoff for the range considered (from 0 to $2 \mathrm{~mm}$ ) to within $2 \%$ for the laboratory-scale model.

The relationship between $A$ and $W$ for different contrasts of resistivity $\left(R_{x o} / R_{m}\right)$ was then simulated for the laboratory scale (Figure 2). In the range of the fracture apertures $(0.1$ to $1 \mathrm{~mm})$ and contrasts of resistivity $(1 / 100$ to $1 / 10,000)$ considered, the relationship was found to be linear, as described by equation 1 . For larger apertures (depending on the resistivity contrast), the relationship starts deviating from linearity and thus from equation 1 . Parameters $b$ and $c$ for our laboratory configuration can be determined by fitting the results of the numerical simulations with equation 1 .

\section{Optimizing the laboratory setup}

Numerical modeling is a helpful tool for planning the laboratory setup because the electric response can be studied for different setup configurations to determine the most suitable laboratory configuration. Practical constraints make it difficult to work with very large samples in the laboratory. Therefore, small-scale samples (blocks of $150 \times 150 \times 150 \mathrm{~mm}$ ) were selected because the numerical simulations verified that the model (equation 1) is valid also at the small scale, at least for fracture apertures not larger than $1 \mathrm{~mm}$, above which the relationship $A$ versus $W$ deviates from linearity. It is important to use samples of the same size to be consistent and have comparable results.

Because it is not possible to perform laboratory experiments with the entire tool, the actual configuration will differ from the borehole configuration. In fact, the laboratory configuration simulates the near-wellbore response of the tool to the presence of a fracture, and therefore the current densities and lines must approximate those in the borehole. Therefore, the numerical simulations were used to optimize the laboratory configurations, and a particularly sensitive aspect was found to be the position and dimension of the return electrode. An electrode plate covering the full block faces behind the fracture gives an electric response with behavior similar to the results reported by Luthi and Souhaité (1990). Based on these numerical simulations, the return electrode for the laboratory experiments was chosen as a copper plate covering the full surface behind the blocks.

\section{LABORATORY SETUP}

\section{Description}

A diagram of the implemented laboratory setup and its connections is shown in Figure 3. In this system, a constant potential difference is applied across the sample between the measuring pad and a return electrode. The pad is attached to a carriage, which is fixed to a rigid frame directly anchored to the supporting table (Figure 4). Frame bolts can be used to adjust the height and inclination of the carriage. The movement and speed of the carriage can be controlled from the computer with a stepping motor attached to the side of the carriage. To determine precisely the relative position of the pad, a sensor was installed on top of the carriage and connected directly to the pad. The central part of the measuring electrode on the pad is in contact with the top surface of the sample, and the return electrode is a copper plate placed at the bottom of the rock samples. The setup is installed in a climate room to perform measurement at a constant temperature $\left(25 \pm 0.5^{\circ} \mathrm{C}\right)$ because the conductivity of saline solutions is temperature dependent.

\section{Data acquisition}

All parameters of equation 1 (i.e., $A, W, R_{m}$, and $R_{x o}$ ) are controlled and determined precisely during the experiments. The additional integrated current is obtained through data processing from the button current measured by the pad. A function generator applies a potential difference between the pad and the return electrode. The computer controls the function generator $(0$ to $7 \mathrm{~V})$, the pad

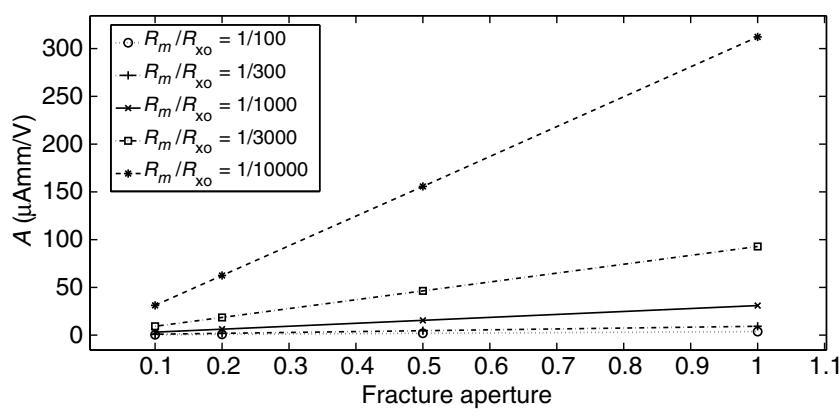

Figure 2. Computed values of integrated additional current $(A)$ for four different fracture apertures $(W=0.1$ to $1 \mathrm{~mm})$ and five different resistivity contrasts $\left(R_{m} / R_{x o}=1 / 100\right.$ to $\left.1 / 10,000\right)$ at zero standoff.

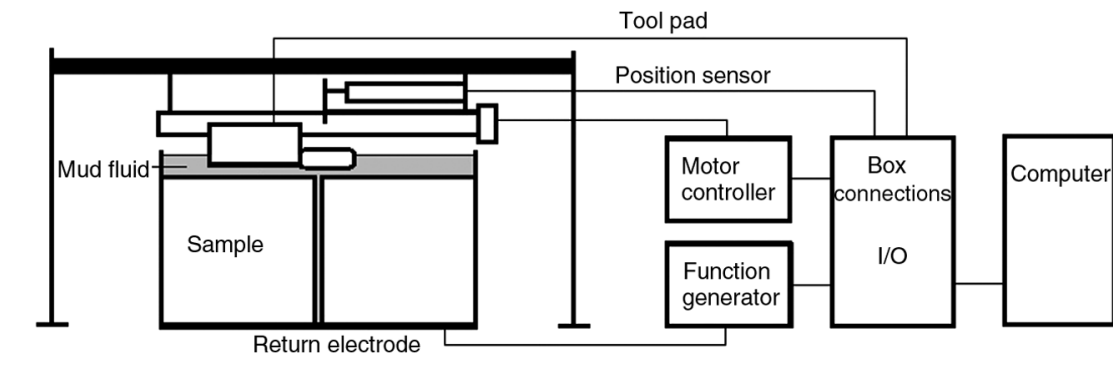

Figure 3. Schematic diagram of the experimental setup for fracture characterization. 
position (0 to $150 \mathrm{~mm})$, and the pad velocity $(0.005$ to $0.11 \mathrm{~mm} / \mathrm{s})$. During a measuring cycle, the pad moves across the fracture and the current of the pad button is measured and recorded (accuracy of $\pm 1 \mu \mathrm{A})$. The different fluid resistivities are obtained with salt $(\mathrm{NaCl})$ and distilled water and determined using a conductivity meter (LF 340). To measure the resistivity of the rock samples, a fourelectrode measuring device was designed with two plates placed on opposite faces of the sample. While the current is injected through the block, the potential across the sample is measured by two electrode stripes glued on each plate and insulated from the current electrodes. The device has been calibrated and was found to work properly for resistances up to $1.5 \mathrm{M} \Omega$, with an error of $\pm 2 \%$. The fracture apertures are set using spacers of known thicknesses between the two blocks. To determine the actual value of $W$ and to verify that it is constant along the fracture, a digital microscope (Dino-Lite Pro AM-413T) is used to acquire high-resolution digital pictures at several locations along the fracture whose apertures can then be determined with an accuracy of $13 \mu \mathrm{m}$ for pictures taken at $60 \times$ magnification typically used during the experiments.

\section{Calibration of the setup}

A relevant issue of fracture characterization with laboratory experiments is the wide range of currents that the tool pad encounters. When the pad is far from the fracture, the high-sample resistance produces a very small current (in the range $0.1 \mathrm{~mA}$ ), whereas near the conductive fracture, the resistance decreases significantly. Therefore, to cover several orders of magnitude of the measured resistance, three internal switches are added to the system to amplify or divide the potential depending on the value of resistance encountered. The voltage is divided by 50 for conductive paths $(R<14 \mathrm{k} \Omega)$ between the pad and the return electrode, whereas it is amplified by 10 for resistances above $140 \mathrm{k} \Omega$.

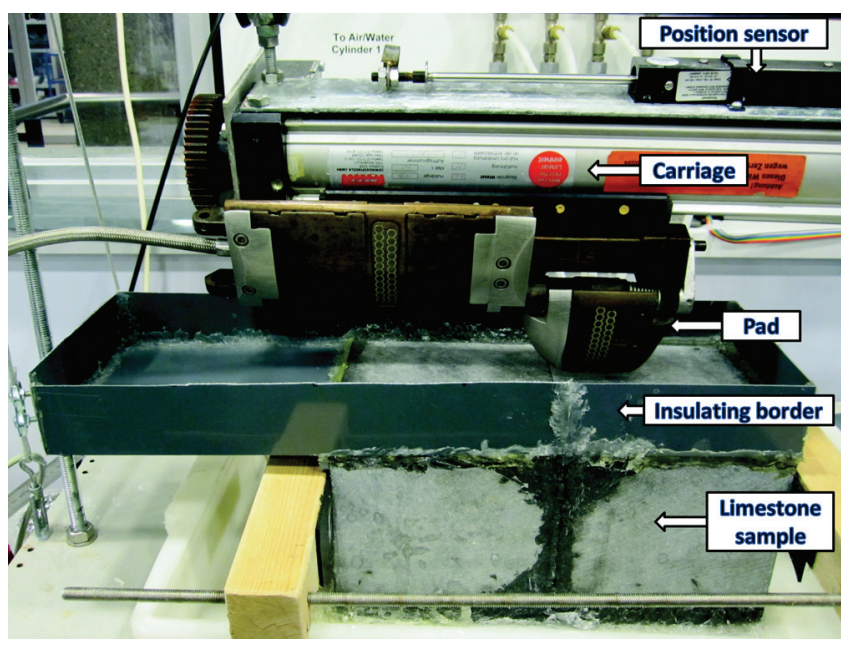

Figure 4. The tool and the sample assembly before the experiments. The tool pad is mounted on a carriage, and its movement is controlled with a motor and a position sensor. The upper part of the tool is a flap that can be turned with pressure on the top surface of the sample and thus it can ensure a good contact between the sample and the buttons. The sample consists of two limestone blocks pressed against each other with spacers in between. The blocks are sealed with silicon to allow the fluid only on the top of the sample and in the fracture.
The range of resistances measured by the setup was determined by calibrating the electric system with reference resistors from $100 \Omega$ to $12 \mathrm{M} \Omega$, which were connected between the measuring pad button and the return electrode. The calibration results showed that the resistance is measured accurately (below $2 \%$ error) up to $2 \mathrm{M} \Omega$, whereas it deviates progressively from the expected values above $2 \mathrm{M} \Omega$. Even for relatively conductive samples (in the $\mathrm{k} \Omega \mathrm{m}$ range), the resistance between the pad button and the return electrode can be larger than $2 \mathrm{M} \Omega$. Therefore, the measured resistance should be corrected based on the results of the calibration. Because the difference between the measured resistance and the correct resistance increases regularly, it is possible to derive a calibration factor $k$ in the form of a function to be applied to measured resistances $R$ above $2 \mathrm{M} \Omega$.

The corrected resistance $\left(R_{c}\right)$ can be thus obtained from the relationship

$$
R_{c}=k(R)
$$

where $k$ is a function of $R$ that, according to the calibration data, can be written as

$$
k=a_{1} R^{a_{2}}+a_{3} .
$$

By fitting of the calibration data with equation 4 , it is possible to derive the calibration parameters $a_{1}=55.4, a_{2}=0.735$, and $a_{3}=-3.45 \cdot 10^{5}$. These values are then used to correct the experimental data and compute the correct resistances from measured resistances higher than $2 \mathrm{M} \Omega$.

\section{METHODS}

\section{Sample selection}

Ideally, the rock material to be used as a sample for the laboratory experiments should be nonporous and impermeable to maintain a constant and uniform resistivity $R_{x o}$ for the duration of the experiments, i.e., to avoid fluid invasion. Moreover, it should have a resistivity in the range of 100 to $10,000 \Omega \mathrm{m}$ and be relatively homogeneous throughout its volume.

Based on these considerations, Irish blue limestone blocks were selected for the experiments. This type of limestone is of Carboniferous age and is nonporous, with a high percentage of calcite (in excess of 96\%) and the remainder being clay and organic material, which accounts for its relatively dark color. It is sufficiently homogeneous, and the presence of dispersed clay particles makes it relatively conductive. The resistivity of the Irish limestone samples measured with the four-electrode resistivity device mentioned above showed a value of $2400 \Omega \mathrm{m}$. Other samples consisting of saturated cement at different resistivities were also tested. However, their relatively high conductivity created too many spurious currents for the results to be a good simulation of the borehole situation. This was due to the high permeability of the cement blocks, which resulted in a value of $R_{x o}$ not constant over time and for different experiments.

\section{Measurement procedure}

To validate equation 1 with the tool experiments, the fracture response must be determined for a wide range of fracture apertures 
and resistivity contrasts. Experiments are conducted on Irish limestone samples for five different saline solutions with corresponding resistivity contrasts, as shown in Table 1 . For each of the five saline solutions, four different fracture apertures and four standoffs are investigated, for a total of 80 experiments. A view of the pad and the sample is shown in Figure 4. Two limestone blocks are pressed against each other with spacers of desired thickness in between. It was experienced that - even for polished and finished rocks samples - it was difficult to produce uniform apertures smaller than $100 \mu \mathrm{m}$. An insulating border is sealed around the two blocks with glue and silicon to allow the fluid only on the top of the sample and in the fracture. The pad is lowered until perfect contact with the top face of the sample is established (zero standoff); to create standoff, a spacer is placed between the pad and the surface of the sample. The fracture and the top surface are then filled with the saline solution, and the exact value of $R_{m}$ is monitored continuously during the experiments with a conductivity meter to determine a precise value for the calculations. For each fracture aperture, several repeat scans are performed at a pad velocity of $0.05 \mathrm{~mm} / \mathrm{s}$, and the current of a central button on the pad is measured. For each test, the values of the applied voltage and the measured current as a function of pad position are automatically stored in a file for data processing. After these measurements, the saline solution in the fracture is displaced, and the sample is dried with pressurized air; the fracture is then refilled with a solution of higher conductivity $R_{m}$ until all five saline solutions (Table 1) are used. Subsequently, the two blocks are opened, the silicon is removed, and the resistivity is measured before preparing a new fracture aperture.

The current values are corrected according to the calibration results. Pad positions are converted to a distance relative to the fracture position. The integrated additional currents produced by the fracture response are then calculated with equation 2 for different values of $R_{m} / R_{x o}$, and they are processed to determine the tool parameters $b$ and $c$. For this purpose, equation 1 can be rewritten in the following way:

$$
\frac{W}{A R_{x o}}=c\left(\frac{R_{m}}{R_{x o}}\right)^{b} .
$$

By fitting the experimental data with equation 5, the values of the tool parameters corresponding to the laboratory configuration are obtained.

\section{RESULTS}

For each of the total of 80 experiments for the selected apertures, resistivity contrasts, and standoffs, several scans were run, resulting in 219 tests. Figure 5 shows the effect of standoff on the measured current. For a fixed aperture and mud resistivity, the button currents decrease in their peaks but widen in their shape with increasing standoff. However, the overall value of $A$ is similar for all the curves, and it varies from 4.8 to $5.1 \mu \mathrm{Amm} / \mathrm{V}$.

Figure 6 shows the whole set of experiments. As with the numerical simulations, the experimental data fit the model expressed by equation 5. The derived tool parameters obtained from the best fit of the laboratory experiments to the equation with the leastsquares method are $b=0.86$ and $c=2.49 \mathrm{~mm}^{-1}$. These values differ from the numerical ones $\left(b=0.98\right.$ and $\left.c=10.98 \mathrm{~mm}^{-1}\right)$ be- cause of some geometric differences in the simplification of the setup, but because the numerical and experimental data fit the model, the linearity and general validity of equation 5 is confirmed.

Subsequently, equation 1 was used to predict the fracture aperture of the limestone samples using optimal values of the tool parameters, obtained as the average of the experimental results $(b=0.86$ and $c=2.49 \mathrm{~mm}^{-1}$ ). Table 2 shows the derived apertures for the samples considered. The difference between the derived apertures and the estimated apertures from the digital microscope is less than $10 \%$ for all the fracture apertures, and thus the model proves to be effective in estimating $W$ from calculated values of $A$. The coefficient of variation (standard deviation divided by the mean) increases with smaller fractures from $8.6 \%(W=0.9 \mathrm{~mm})$ to $44 \%(W=0.1)$.

\section{DISCUSSION}

Equation 1 models the fracture response with an FMI tool; it was derived numerically, and it expresses a linear relationship between

Table 1. Saline solutions resistivities and resistivity contrasts used for the experiments.

\begin{tabular}{cccccc}
$R_{m} / R_{x o}$ & $1 / 120$ & $1 / 300$ & $1 / 1000$ & $1 / 3000$ & $1 / 10,000$ \\
\hline$R_{m}(\mathrm{~m})$ & 20 & 8 & 2.4 & 0.8 & 0.24 \\
\hline
\end{tabular}

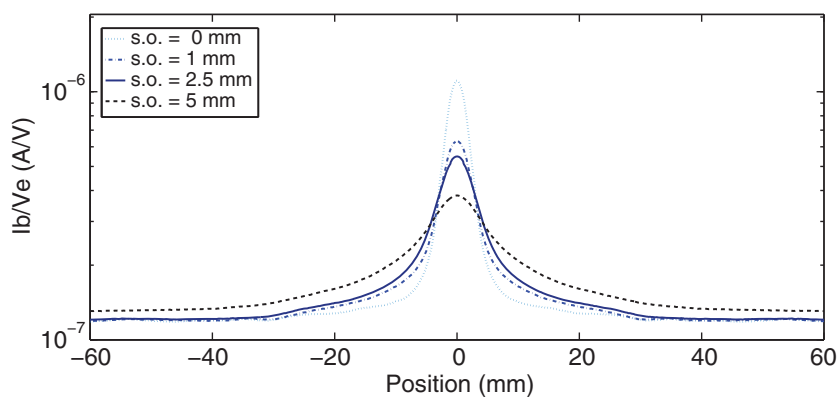

Figure 5. Experimental data at a fixed aperture $(W=0.1 \mathrm{~mm})$ and resistivity contrast $(1 / 1000)$ for different standoffs $(0$ to $5 \mathrm{~mm})$.

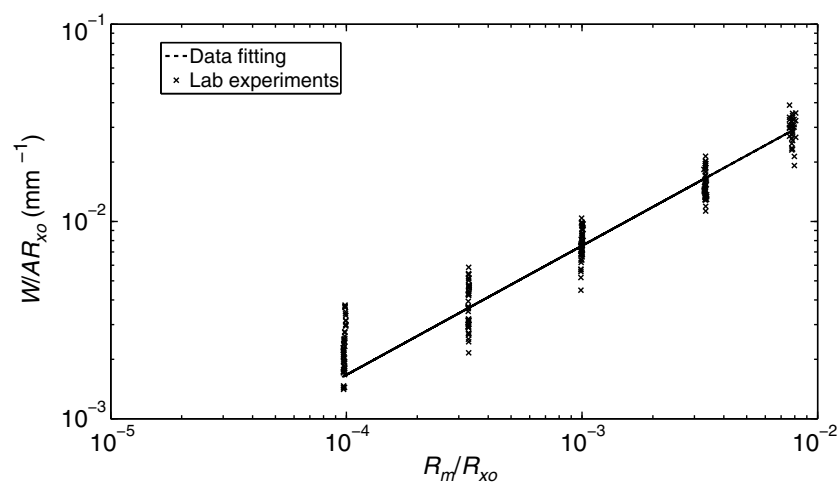

Figure 6. Fitting of experimental data with equation 5 . The whole set includes 219 experiments with varying apertures $(0.1$ to $0.9 \mathrm{~mm})$, resistivity contrasts $(1 / 100$ to $1 / 10,000)$, and standoffs (0 to $5 \mathrm{~mm}$ ). 
the fracture aperture and the measured integrated additional current. The objective of this study was to validate this relationship for such a tool with laboratory experiments. The experimental results and the numerical simulations proved that the linearity of the relationship is also valid for tool experiments at the laboratory scale. The measured integrated additional current is linearly proportional to the fracture aperture, whereas an increase of the mud resistivity results in a lower value of $A$. The experiments confirmed that the standoff does not significantly affect the value of $A$, but it changes only the amplitude of the peak and the shape of the measured current curve. The tool pad proved to work properly also for large standoffs $(5 \mathrm{~mm})$, but the fracture is detected at a further distance, and thus a longer path is necessary to compute the overall value of $A$. The tool parameters $b$ and $c$ have been derived by fitting of the experiments with the model. Values of $b$ are similar to the ones obtained from borehole simulations (Luthi and Souhaité, 1990), whereas $c$ is about half the value. Coefficient $c$ is proportional to a geometric factor $k$ (Doll, 1949), which relates the resistance measured by the tool to the actual formation resistivity $R_{x o}$, and it is a function of the borehole and tool configuration. Therefore, variations of $c$ are due to differences in the geometric configuration of the laboratory setup compared with the borehole configuration and with variations in the tool geometry from the tool studied by Luthi and Souhaite (1990) and the tool in this study. Higher values of tool parameters obtained with numerical simulations can be attributed to geometric simplification in the design of the setup in the numerical modeling.

The fracture aperture of the limestone samples was determined with equation 1 based on the tool parameters derived from the experiments. It was found that the digital microscope and equation 1 estimates of $W$ differed by a maximum of $10 \%$ for all the experiments performed. Therefore, the FMI is an effective tool to estimate fracture apertures from button current data independent of standoff, when the formation and mud resistivity are known. However, it is essential to use the exact tool parameters for the configuration considered. In this paper, we determined tool parameters for the laboratory scale, whereas for borehole data, we expect that the correct values are the ones presented by Luthi and Souhaité (1990).

A limitation of these experiments is that only ideal fractures were considered (perpendicular to the measuring pad and with parallel faces). For more realistic fractures (nonplanar and nonparallel), the dip angle and tortuosity may also be considered because the flow and transport properties are influenced by the roughness of the fracture walls and the spatial variations of their local apertures (e.g., Méheust and Schmittbuhl 2000; Matsuki et al., 2006; Watanabe et al., 2008). Electric measurements can provide information about apertures in rough fractures (Boschan et al., 2011), as with

Table 2. Values of fracture apertures of each sample obtained with the digital microscope and derived from the laboratory experiments.

$W$ microscope (mm)

$W$ lab experiments $(\mathrm{mm})$
0.10
$0.09 \pm 0.04$
0.20
$0.18 \pm 0.05$
0.50
$0.47 \pm 0.11$
0.90
$0.81 \pm 0.07$

increasing tortuosity or roughness of the walls, a lower electric aperture is expected (Brown, 1989). This would lead to a lower value of $A$ with respect to fractures with parallel walls, and equation 1 should be modified adding a tortuosity factor.

Another limitation for the applicability of the model concerns formations with a very shallow or no invasion. Furthermore, the model was verified only within a specific range of apertures and resistivity contrasts and the behavior at large standoffs was not tested. Compared with the borehole simulations (Luthi and Souhaité, 1990), we extended the range of apertures up to $1 \mathrm{~mm}$, but for the samples used, it was not possible to exceed the resistivity contrasts beyond 1/10,000 because of salt saturation of the brine solution. For larger apertures, a deviation from linearity of the model is expected, as was indicated by the numerical modeling.

\section{SUMMARY AND CONCLUSIONS}

The full-bore FMI is a tool that can be used to characterize fractures. Previously performed numerical simulations showed a linear relationship between the measured integrated additional current $A$ and the aperture $W$ of open, mud-filled fractures in a borehole. To validate this relationship with experiments, a laboratory setup was designed and built. It allowed for performing electric scans of fractured blocks under controlled conditions of temperature, fracture aperture, pad position, and formation, as well as mud resistivity. Three-dimensional numerical modeling of the experiments was used to select the appropriate laboratory configuration, specifically of the return electrode position and sample size, and to study the tool's fracture response at the laboratory scale to validate the experimental results. Experiments were conducted for several fracture apertures (0.1 to $0.9 \mathrm{~mm})$, resistivity contrasts (1/100 to $1 /$ $10,000)$, and standoffs ( 0 to $5 \mathrm{~mm}$ ) on selected samples (Irish blue limestone). The results confirmed the linearity between $W$ and $A$ for the samples considered. Average tool parameters for the specific tool configurations were derived and used to calculate the fracture aperture of each sample. Numerical simulations reproduced the experimental results for the same fracture properties and could be compared with the previous borehole simulations. Differences in the tool parameters are attributed to differences in electrode contacts and length scales of the setup.

\section{ACKNOWLEDGMENTS}

This work has been supported and carried out in collaboration with Études et Productions Schlumberger, Clamart, France. In particular, the authors wish to thank P. Cheung and I. Dubourg for the fruitful discussions. We thank K. van Beek and R. van Leeuwen of TU Delft for the contribution in the development of the electronics of the setup, and we thank A. Hemstede (TU Delft) for the logistics and mechanical connections of the setup.

\section{REFERENCES}

Boschan, A., I. Ippolito, R. Chertcoff, J. P. Hulin, and H. Auradou, 2011, Characterization of fracture aperture field heterogeneity by electrical resistance measurement: Journal of Contaminant Hydrology, 123, 65-74, doi: 10.1016/j.jconhyd.2010.12.007.

Bratton, T., D. V. Canh, N. Van Que, N. V. Duc, P. Gillespie, D. Hunt, B. Li, R. Marcinew, R. Satyaki, B. Montaron, R. Nelson, D. Schoderbek, and L. Sonneland, 2006, The nature of naturally fractured reservoirs: Oilfield Review, 18, 4-23. 
Brown, S. R, 1987, Fluid flow through rock joints: The effect of surface roughness: Journal of Geophysical Research, 92, 1337-1347, doi: 10 $.1029 / \mathrm{JB} 092 \mathrm{iB} 02 \mathrm{p} 01337$.

Brown, S. R., 1989, Transport of fluid and electric current through a single fracture: Journal of Geophysical Research, 94, 9429-9438, doi: 10.1029/ JB094iB07p09429.

Brown, S. R. and C. H. Scholz, 1986, Closure of rock joints: Journal of Geophysical Research, 91, 4939-4948, doi: 10.1029/ JB091iB05p04939.

Doll, H. G., 1949, Introduction to induction logging and application to wells drilled with oil base mud: Journal of Petroleum Technology, 1, 148-162, doi: 10.2118/949148-G.

Ekstrom, M. P., C. Dahan, M.-Y. Chen, P. Lloyd, and D. Rossi, 1987, Formation imaging with microelectrical scanning arrays: The Log Analyst, 28, 294-306.

Gangi, A. F., 1978, Variation of whole and fractured porous rock permeability with confining pressure: International Journal of Rock Mechanics and Mining Sciences and Geomechanics Abstracts, 15, 249-257, doi: 10 $.1016 / 0148-9062(78) 90957-9$.

Laronga, R., G. T. Lozada, F. M. Perez, P. Cheung, S. M. Hansen, and A. M. Rosas, 2011, A high-definition approach to formation imaging in wells drilled with nonconductive muds: Presented at the SPWLA 52nd Annual Logging Symposium.
Luthi, S. M., 2001, Geological well logs — Their use in reservoir modeling: Springer Verlag.

Luthi, S. M., and P. Souhaité, 1990, Fracture apertures from electrical borehole scans: Geophysics, 55, 821-833, doi: 10.1190/1.1442896.

Matsuki, K., Y. Chida, K. Sakaguchi, and P. W. J. Glover, 2006, Size effect on aperture and permeability of a fracture as estimated in large synthetic fractures: International Journal of Rock Mechanics and Mining Science, 43, 726-755, doi: 10.1016/j.ijrmms.2005.12.001.

Méheust, Y., and J. Schmittbuhl, 2000, Flow enhancement of a rough fracture: Geophysical Research Letters, 27, 2989-2992, doi: 10.1029/1999GL008464.

Pezard, P. A., and S. M. Luthi, 1988, Borehole electrical images in the basement of the Cajon Pass scientific drillhole; fracture identification and tectonic implications: Geophysical Research Letters, 15, 1017-1020, doi: 10 1029/GL015i009p01017.

Rajabi, M., S. Sherkati, B. Bohloli, and M. Tingay, 2010, Subsurface fracture analysis and determination of in-situ stress direction using FMI logs: An example from the Santonian carbonates (Ilam Formation) in the Abadan Plain, Iran: Tectonophysics, 492, 192-200, doi: 10.1016/j.tecto.2010.06.014.

Watanabe, N., N. Hirano, and N. Tsuchiya, 2008, Determination of aperture structure and fluid flow in a rock fracture by high-resolution numerical modeling on the basis of a flow-through experiment under confining pressure: Water Resources Research, 44, W06412, doi: 10.1029/ 2006WR005411. 